\title{
Investigation of Noise Suppression, Pulse Intensity and Chirp of an Actively Mode-Locked Semiconductor Fiber Ring Laser
}

\author{
Ivan Evans ${ }^{1}$, Colm O’Riordan ${ }^{1}$, Michael J. Connelly ${ }^{1}$, Liam P. Barry ${ }^{2}$, Aisling M. \\ Clark $^{2}$, Prince Anandarajah ${ }^{2}$. \\ (1) Optical Communications Research Group, Department of Electronic and \\ Computer Engineering, University of Limerick, Limerick, IRELAND. E-mail: \\ michael.connelly@ul.ie
}

(2) Radio and Optical Communications Research Group, Research Institute for Networks and Telecommunications Engineering, Dublin City University,

Dublin 9, IRELAND. E-mail: liam.barry@dcu.ie

\begin{abstract}
An actively mode-locked fiber ring laser that utilizes an EDFA and semiconductor optical amplifier (SOA) is presented. The SOA enhances noise suppression. Active mode-locking was demonstrated at repetition rates up to $20 \mathrm{GHz}$. The effects of intra-cavity power and SOA current on noise suppression are studied for two types of commercially available SOA. The Frequency Resolved Optical Gating technique is used to characterize the output pulse stream intensity and frequency chirp.
\end{abstract}

Index terms - Ring laser, mode-locking, semiconductor optical amplifier, frequency resolved optical gating, optical pulse measurements.

\section{INTRODUCTION}

Harmonically mode-locked fiber ring lasers have the capability to produce stable, tunable, relatively high power and high repetition rate short pulse outputs. The applications for such sources range from sources for optical TDM, communications component characterization, sensing, optical sampling and optical comb generation.

Several challenges must be first overcome in order to produce a robust EDFA based mode-locked laser source. A combination of factors such as long cavity length, long lifetime of the erbium gain medium and poor discrimination between the dominant and side modes mean that such harmonically mode-locked lasers are susceptible to environmental fluctuations. This can result in noisy operation. A variety of solutions to this problem have been demonstrated, including configurations utilizing regenerative mode-locking and Fabry-Perot intracavity filters [1,2]

A solution to the supermode noise problem has been demonstrated which incorporates a semiconductor optical amplifier (SOA) in the laser cavity [3]. The addition of the SOA, when biased above transparency, suppresses supermode noise and relaxation oscillations caused by the EDFA. This technique has been further demonstrated in a dispersion tuned mode-locked laser which operated at 6 and $10 \mathrm{GHz}[4,5]$. In this laser the RF side mode suppression was determined as a function of the SOA current. The noise suppression in a narrow linewidth CW EDFA ring laser was also shown to be improved by the addition of an intra-cavity SOA [6]. Side mode suppression ratios in excess of $80 \mathrm{~dB}$ were achieved in a $1 \mathrm{GHz}$ repetition rate $\mathrm{SOA}$ based mode-locked laser under certain operating conditions [7]. The single side band phase noise and related jitter was also shown to vary with the SOA bias current. If the device was 
operated without a band pass filter a significant degradation in phase noise was observed. The nature of the noise suppression has been attributed to gain saturation in the SOA and has been the subject of a theoretical analysis in $[8,9]$.

The incorporation of a SOA in a harmonically mode-locked laser brings many advantages including super mode noise suppression and the facility to actively modulate the SOA.

In this paper the noise suppression due to two different types of commercial SOA used in an actively mode-locked semiconductor fiber laser is characterized. Frequency Resolved Optical Gating (FROG) measurements are used to determine the laser output pulsewidth and chirp as a function of SOA current and input power at repetition rates up to $20 \mathrm{GHz}$.

\section{ACTIVELY MODE-LOCKED SEMICONDUCTOR FIBER LASER}

\section{A. Experimental Setup}

The laser is shown in Fig.1. An SOA and EDFA provide the optical gain in the fiber ring. The EDFA has a small signal gain of $30 \mathrm{~dB}$ and a saturation output power of 10 $\mathrm{dBm}$. A Lithium Niobate amplitude modulator allows the laser to be actively modelocked. The polarization of the laser light is controlled by two polarization controllers. The power entering the SOA is controlled by an attenuator. The lasing wavelength is controlled by a filter placed after the EDFA. In order to maximize the output power from the laser the lossiest element in the ring, the intensity modulator, is positioned before the EDFA. Light is coupled from the ring using a $3 \mathrm{~dB}$ coupler. The cavity length is $42 \mathrm{~m}$, which corresponds to a fundamental frequency of $5 \mathrm{MHz}$.

Two types of commercial SOA were used in the experiment. SOA-1 (optical preamplifier OPA-C from Kamelian) has a small signal gain of $22 \mathrm{~dB}$, a saturation output power of $12.4 \mathrm{dBm}$ and a gain recovery time of $60 \mathrm{ps}$. SOA-2 (SOA-NL-OEC1550 from CIP) has a large optical nonlinearity, a small signal gain of $25 \mathrm{~dB}$, a saturation output power of $4.5 \mathrm{dBm}$ and a short saturated gain recovery time of $20 \mathrm{ps}$. The small-signal gain versus the ratio of the current to transparency current characteristics of the SOAs are shown in Fig. 2(a). The transparency currents $\left(I_{t}\right)$ of the SOAs at $1550 \mathrm{~nm}$ are $22.5 \mathrm{~mA}$ and $56.0 \mathrm{~mA}$ for SOA-1 and SOA-2 respectively. Typical gain versus output power characteristics of both SOAs are shown in Fig. 2(b). The slope of the gain versus output power characteristic in the saturation regime is much greater for the CIP device compared to the Kamelian device. This means that saturation effects are more easily achieved in the CIP device.

\section{B. Noise Suppression}

The supermode noise usually present in harmonically mode-locked lasers can be quantified by measuring the ratio of the intensity of the signal centre frequency (equal to the pulse repetition frequency) and the highest intensity of adjacent undesired harmonics using an r.f. spectrum analyzer. Fig. 3(a) shows this Supermode Suppression Ratio (SSR) versus the bias current normalized to the transparency current. As expected, both SOAs show similar trends with the SSR increasing with bias current. The steeper slope of this characteristic for SOA-2 is attributed to the fact that its gain versus $I / I_{t}$ characteristic, as shown in Fig. 2(a), also exhibits a steeper slope compared to that for SOA-1. For drive currents around and above transparency 
substantial noise suppression, and stable pulse trains result. To maximize noise suppression the SOA should be operated in the saturated regime.

Fig. 3(b) shows that as the SOA input power is increased the SSR increases. This results in an improvement in the quality of the output pulse train as shown in Fig. 4 for the laser using SOA-2. Similar pulse characteristics were obtained when SOA-1 was used; however the SOA-2 based laser exhibited superior pulse quality over a greater range of SOA input power due to its more favorable SSR characteristics.

In the experiments, the amount of attenuation introduced before the SOA does not affect the laser output power, which remains constant, as the EDFA input power is always greater then the input saturation power. A clear difference is observed between the two SOAs we examined: SOA-2 provides a given level of noise suppression for a much larger range of SOA input powers when compared to the lower gain SOA-1. This is because SOA-2 has a lower saturation output power and steeper gain versus output power characteristic, as shown in Fig. 2(b), compared to that for SOA-1. The measurements demonstrate that the presence of an SOA in a fiber laser only has a significant effect on noise suppression if it is operated in a saturated regime. Consequently the location of the SOA in the ring and the design of the SOA are important factors in the overall performance of the laser.

The laser was mode-locked at frequencies of 1, 2, 5, 10 and $20 \mathrm{GHz}$. Table 1 shows the corresponding measured pulsewidth, bandwidth and corresponding timebandwidth product. Fig. 5 shows a sample optical spectrum when the laser is actively mode-locked at $20 \mathrm{GHz}$. The optical bandwidth is $0.36 \mathrm{~nm}$ and for a corresponding pulsewidth of 12 ps this gives a time-bandwidth product of 0.6 . This clearly indicates that the pulses are not transform limited and contain a large amount of frequency chirp, which is confirmed by FROG measurements described in the next section.

\section{Characterization using Frequency Resolved Optical Gating}

The FROG technique in contrast to the traditional methods of autocorrelation, crosscorrelation and optical spectrum analysis, can be used to completely characterize optical pulses in the spectral and temporal domains with corresponding phase information $[10,11]$. FROG was used to measure the output pulse intensity and chirp from the laser using SOA-2 at a repetition rate of $20 \mathrm{GHz}$. As described above the SSR improves with increasing SOA current and input power. However at lower repetition rates, around $1 \mathrm{GHz}$, significant pulse distortion is observed at the higher SOA bias currents. Figs. 6(a) and 6(b) show the output pulse normalized intensity as a function of bias current and SOA input power. The measured pulsewidth versus bias current and SOA input power are shown in Figs. 7(a) and 7(b) respectively. The pulse broadening and asymmetry increases with bias current and SOA input power. The asymmetry in the pulse profile is because the leading and trailing edges of the pulse experience different dynamic gain. As the SOA input power is increased from -18 $\mathrm{dBm}$ to $5 \mathrm{dBm}$ the pulsewidth increases by $40 \%$. At the higher SOA bias currents and input powers, pulse pedestals are apparent. The level of these pedestals, while much lower than the pulse peak, can cause problems if the laser output is amplified by a second SOA [11].

Figs. 8(a) and 8(b) show the output pulse dynamic chirp with the bias current and SOA input power as parameter respectively. The pulses have a small positive linear chirp (red shift), which is relatively insensitive to changes in the SOA bias and input power. The chirp is mainly generated by the gain saturation-induced refraction index 
changes in the SOA [12]. This has been observed in similar mode-locked semiconductor fiber lasers [5, 13]. Given the linear nature of the chirp it should be straightforward to compress these pulses with dispersion compensating fiber or with linearly chirped fiber gratings. Using such techniques it should be possible to compress the $20 \mathrm{GHz}$ laser pulses from typically 13 ps to around 8 ps (given the level of chirp on the pulses).

\section{CONCLUSIONS}

An actively mode-locked fiber ring laser with a SOA and EDFA gain medium was described and characterized. Two types of SOA were used and the laser was modelocked at 1, 5, 10, and $20 \mathrm{GHz}$. In addition to the trend of increasing SSR with SOA current, we have shown that the SOA input power has a strong effect on the level of noise suppression. Consequently the design of the SOA will influence the level of noise suppression attained for a given SOA input power. A nonlinear SOA with a low saturation output power was compared to a more linear SOA, with a higher saturation output power. The nonlinear SOA provides noise suppression over a larger range of input powers. Our results show that the SOA design and operating conditions are important design factors in such fiber laser systems. The laser was characterized at 20 $\mathrm{GHz}$, a higher frequency than has been reported previously. The effects of SOA current and input power on the mode-locked laser performance were measured using the FROG technique. A clear trend of pulsewidth broadening is shown with increasing SOA input power and current. The measured pulse chirp does not change significantly with SOA input power or current and its linearity make the pulse amenable to compression using well established techniques.

\section{ACKNOWLEDGEMENT}

This work was supported by Science Foundation Ireland Investigator Grant 02/IN1/I42. 


\section{REFERENCES}

[1] M. Nakazawa, E. Yoshida, K. Kimura, "Ultrastable harmonically and regeneratively mode-locked polarization maintaining fiber ring laser", Electron. Lett., Vol. 30, No. 19, pp. 1603-1605, 1994.

[2] G. T. Harvey and L.F. Mollenauer, "Harmonically mode-locked fiber ring laser with an internal Fabry-Perot stabilizer for soliton transmission", Optic Lett., Vol. 18, No 2, pp. 107-109, 1993.

[3] C. Wu and N.K Dutta, "High repetition-rate optical pulse generation using a rational harmonic mode-locked laser", IEEE J. Quantum Electron., Vol. QE-36, No 2, pp. 145-150, 2000.

[4] L. Duan, C. J. K. Richardson, Z. Hu, M. Dagenais and J. Goldhar, "A stable smoothly wavelength-tuneable picosecond pulse generator", IEEE Photon Technol Lett., Vol. 14, No. 6, pp. 840-842, 2002

[5] L. Duan, M. Dagenais and J. Goldhar, "Smoothly wavelength-tunable picosecond pulse generation using a harmonically mode-locked fiber ring laser", J. Lightwave Technol, Vol. 21, No. 4, pp. 930-937, 2003.

[6] Lei Xu, I. Glesk, D. Rand, V. Baby and P. R. Prucnal, "Supression of beating noise of narrow-linewidth erbium-doped fiber ring lasers by use of a semiconductor optical amplifier", Optics Letters, Vol. 28, No 10, pp. 780-782, 2003.

[7] G-R. Lin, M-C- Wu, Y-C Chang and C-L Pan, "Ultrahigh supermode noise suppression ratio of a semiconductor optical amplifier filtered harmonically modelocked erbium doped fiber laser", Optics Express, Vol. 13, No 18, pp. 7215-7224, 2005.

[8] Q. Xu and M. Yao, "Theoretical analyses on short-term stability of semiconductor fiber ring lasers", IEEE J. Quantum Electron., Vo. 39, No. 10, pp. 1260-1265, 2003.

[9] C. Peng, M.Yao, Q. Xu and H. Zhang, "Supression of supermode competitions in SOA fiber mode-locked ring laser," Proc. $15^{\text {th }}$ Annual Meeting of the IEEE Lasers and Electro-Optics Society, pp. 377-378, 2002.

[10] R. Trebino, K.W. Long, D. N. Fittinghoff, J. N. Sweetser, M. A. Krumbugel, and B. A. Richman, "Measuring ultrashort laser pulses in the time-frequency domain using frequency resolved optical gating," Rev. Sci. Instrum., vol. 68, pp. 3277-3295, 1997.

[11] A.M Clarke, M.J. Connelly, P.M. Anandarajah, L.P. Barry and D.A. Reid, "Investigation of pulse pedestal and dynamic chirp formation on picosecond pulses after propagation through a semiconductor optical amplifier," IEEE Photon Tech. Lett., Vol. 17, No. 9, pp. 1800-1802, 2005.

[12] G.P Agrawal, N. A. Olsson, "Self-phase modulation and spectral broadening of optical pulses in Semiconductor laser amplifiers", IEEE J. Quantum Electron., Vol 25, No 11, pp 2297-2306, 1989

[13] D. H. Kim, S. H. Kim, Y. Min, "Relaxation-free harmonically mode-locked semiconductor-fiber ring laser", IEEE Photon Tech. Lett.,, Vol. 11, No. 5, pp 521-523, 1999 


\section{Figure and Table Captions:}

Fig. 1. Experimental actively mode-locked laser system. PC: polarization controller.

Fig. 2. (a) SOA small-signal gain versus the ratio of current to transparency current and (b) Typical SOA small-signal gain versus output power characteristics. The wavelength is $1550 \mathrm{~nm}$.

Fig. 3. (a) SSR versus the bias current normalized to transparency current ( $\mathrm{I}_{\mathrm{t}}$ ). (b) SSR versus SOA input power at comparable currents above transparency: $50 \mathrm{~mA}$ for SOA1 and $80 \mathrm{~mA}$ for SOA2.

Fig. 4. Output pulse trains at $10 \mathrm{GHz}$ repetition rate for SOA input powers of (a) -3 $\mathrm{dBm}$ and (b) $-18 \mathrm{dBm}$ for the ring laser using SOA-2.

Fig. 5. Optical spectrum of $20 \mathrm{GHz}$ actively mode-locked ring laser.

Fig. 6. Output pulse intensity obtained from FROG measurements for the ring laser using SOA-2: (a) SOA currents of $70 \mathrm{~mA}, 100 \mathrm{~mA}$ and $150 \mathrm{~mA}$ for $4 \mathrm{dBm}$ input power and (b) SOA input powers of $4 \mathrm{dBm},-5 \mathrm{dBm}$ and $-15 \mathrm{dBm}$ for an SOA bias current of $120 \mathrm{~mA}$.

Fig. 7. FROG pulsewidth measurements for the ring laser, using SOA-2, at $20 \mathrm{GHz}$ repetition rate as a function of (a) SOA input power at a bias current of $120 \mathrm{~mA}$ and (b) SOA current at $4 \mathrm{dBm}$ input power.

Fig. 8. Output pulse chirp for the ring laser using SOA-2 with (a) $-4 \mathrm{dBm}$ SOA input power and bias current as parameter and (b) $120 \mathrm{~mA}$ bias current and SOA input power as parameter.

Table 1. Pulse pulsewidth, bandwidth and time-bandwidth product for various modulation frequencies for the ring laser using SOA-2. 
Fig. 1

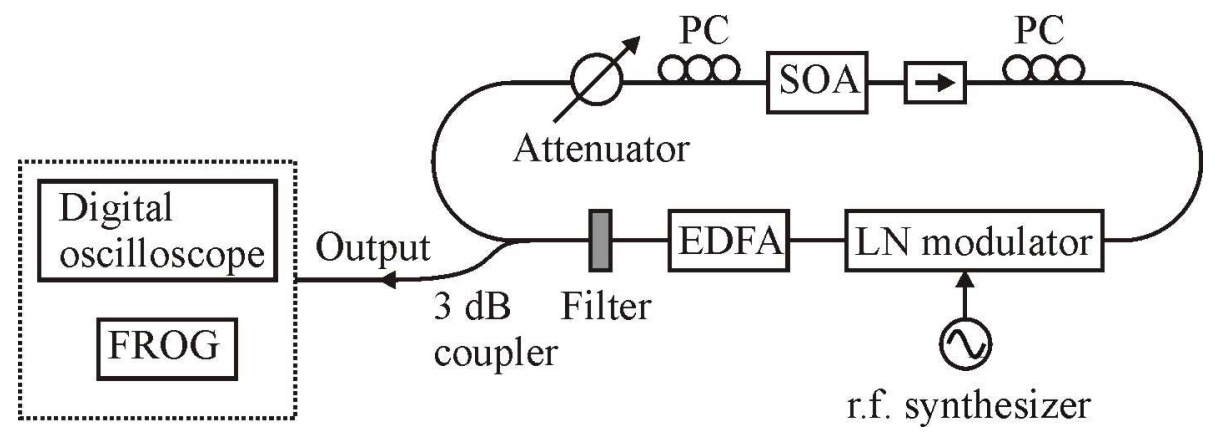


Fig. 2

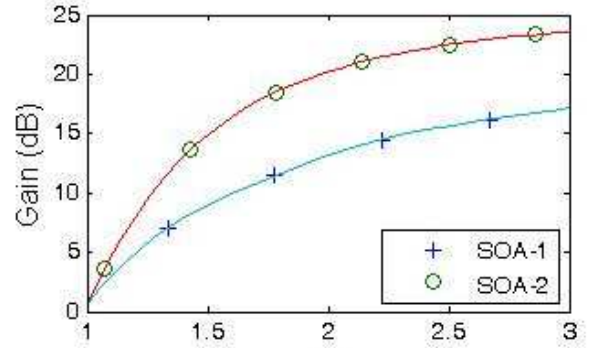

(a)

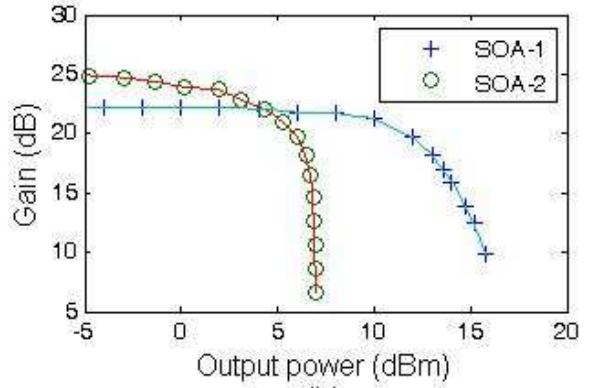

(b) 
Fig. 3

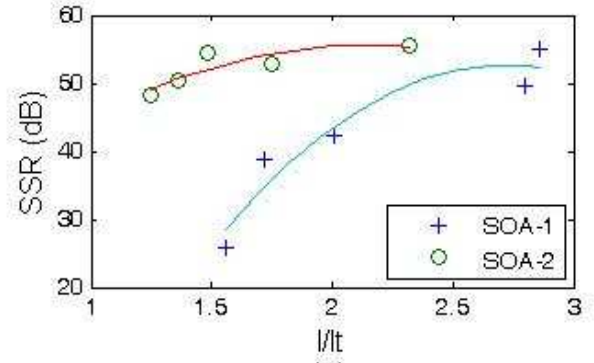

(a)

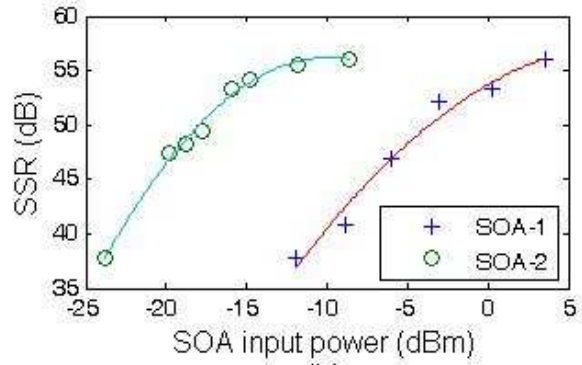

(b) 
Fig. 4

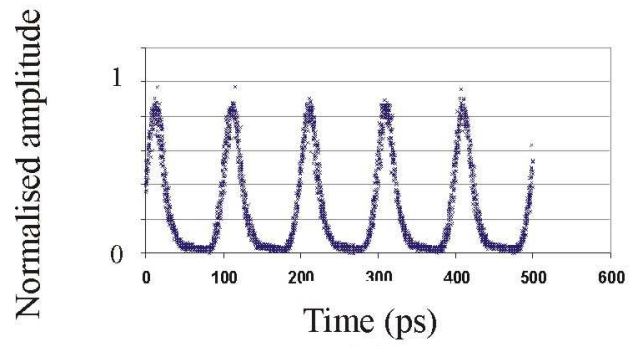

(a)

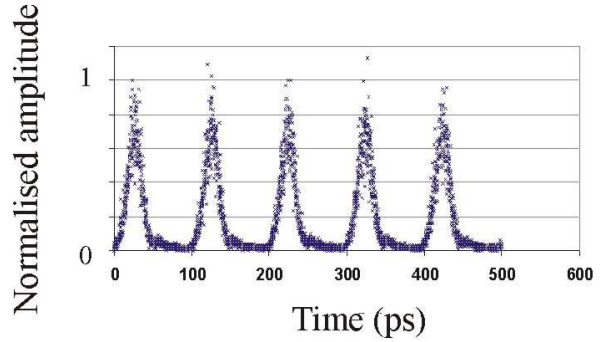

(b) 
Fig. 5

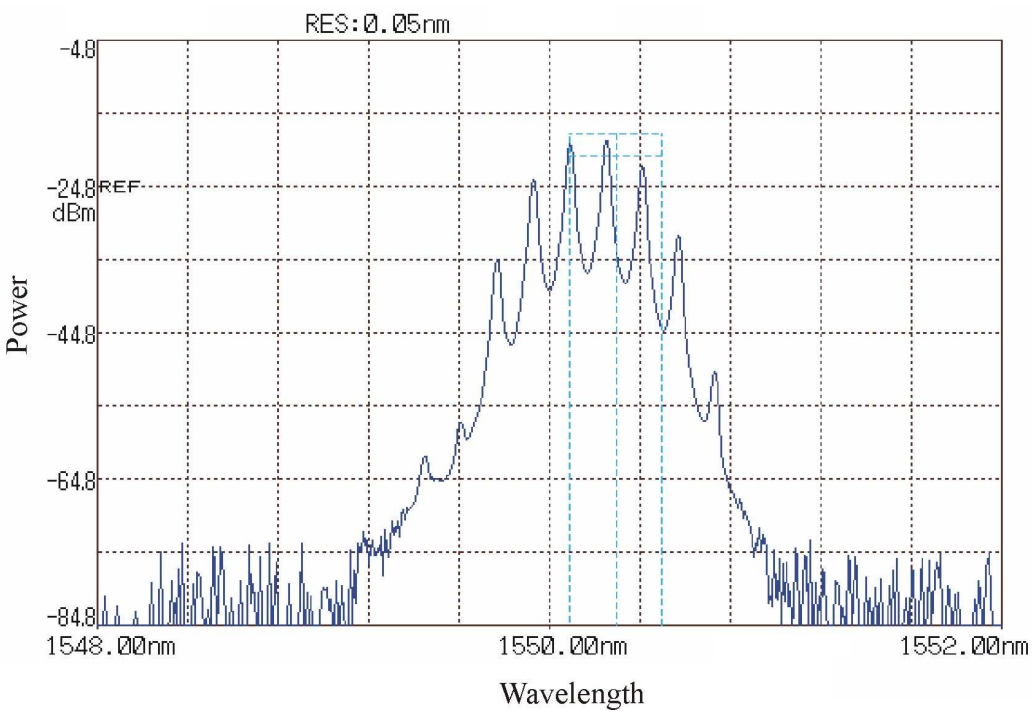


Fig. 6

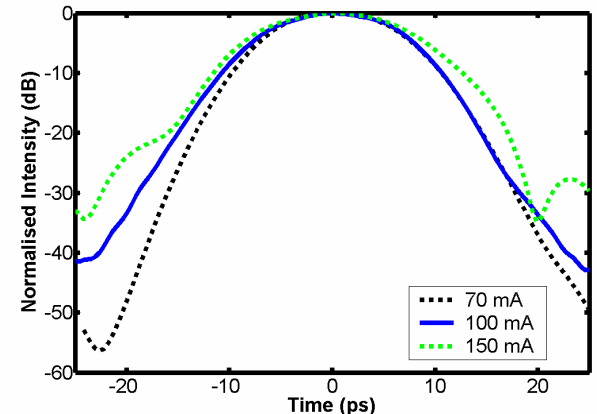

(a)

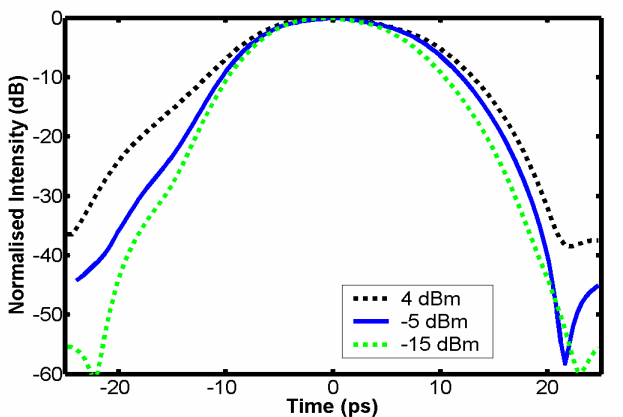

(b) 
Fig. 7

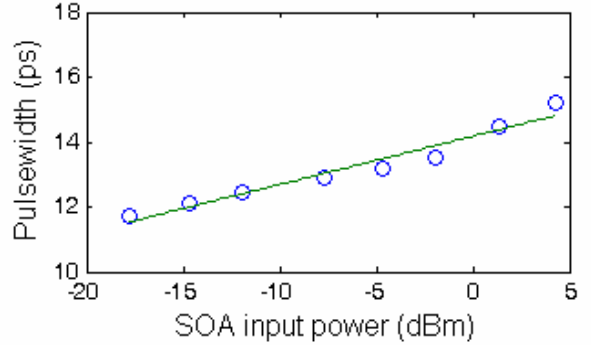

(a)

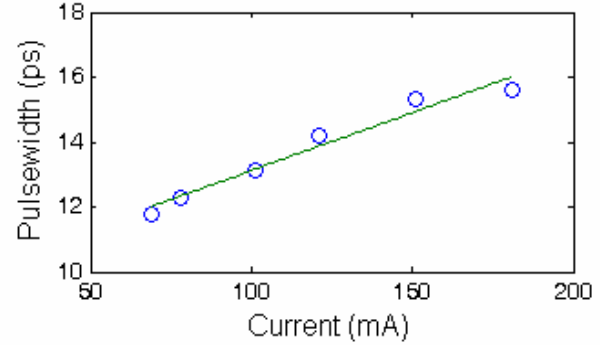

(b) 
Fig. 8

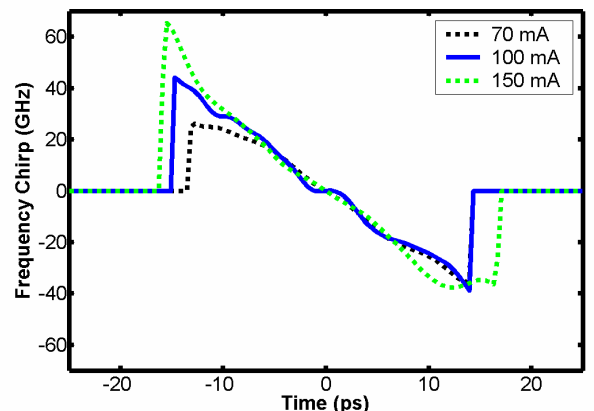

(a)

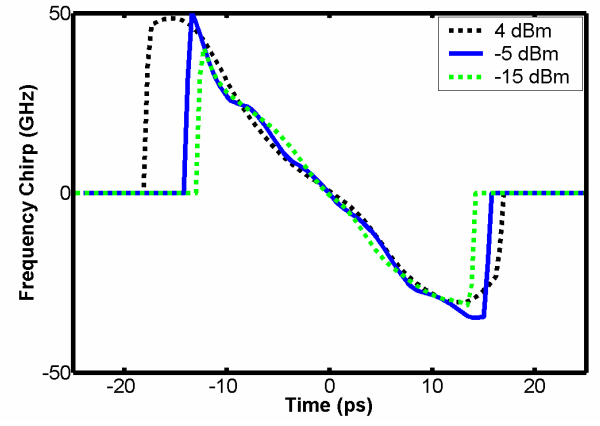

(b) 


\section{Table 1}

\begin{tabular}{|l|l|l|l|}
\hline $\begin{array}{l}\text { Modulation } \\
\text { frequency }(\mathrm{GHz})\end{array}$ & $\begin{array}{l}\text { Full-width at half } \\
\text { maximum } \\
\text { pulsewidth }(\mathrm{ps})\end{array}$ & $\begin{array}{l}\text { 3dB bandwidth } \\
(\mathrm{nm})\end{array}$ & $\begin{array}{l}\text { Time-bandwidth } \\
\text { product }\end{array}$ \\
\hline 1 & 120 & 0.035 & 0.53 \\
\hline 5 & 37 & 0.11 & 0.52 \\
\hline 10 & 25 & 0.16 & 0.52 \\
\hline 20 & 12 & 0.36 & 0.60 \\
\hline
\end{tabular}

